\title{
High Quality Magnetic Oxide Thin Films Prepared via Aqueous Solution Processing
}

Peter Richter, Paul N. Plassmeyer, Julia Harzdorf, Tobias Rüffer, Heinrich Lang, Jana Kalbacova, Nathanael Jöhrmann, Steffen Schulze, Michael Hietschold, Sri Sai Phani Kanth Arekapudi, Manfred Albrecht, Dietrich R.T. Zahn, Catherine J. Page*, and Georgeta Salvan ${ }^{*}$

The X-ray diffractogram of the $800{ }^{\circ} \mathrm{C}$ annealed $\mathrm{CFO}$ film contains all relevant reflection peaks known for spinel type (cubic, Fd$3 \mathrm{~m})$ cobalt ferrite. ${ }^{67}$ The diffractogram compares well to XRD results obtained from other wet-chemically synthesized CFO nanoparticles. $^{68-70}$

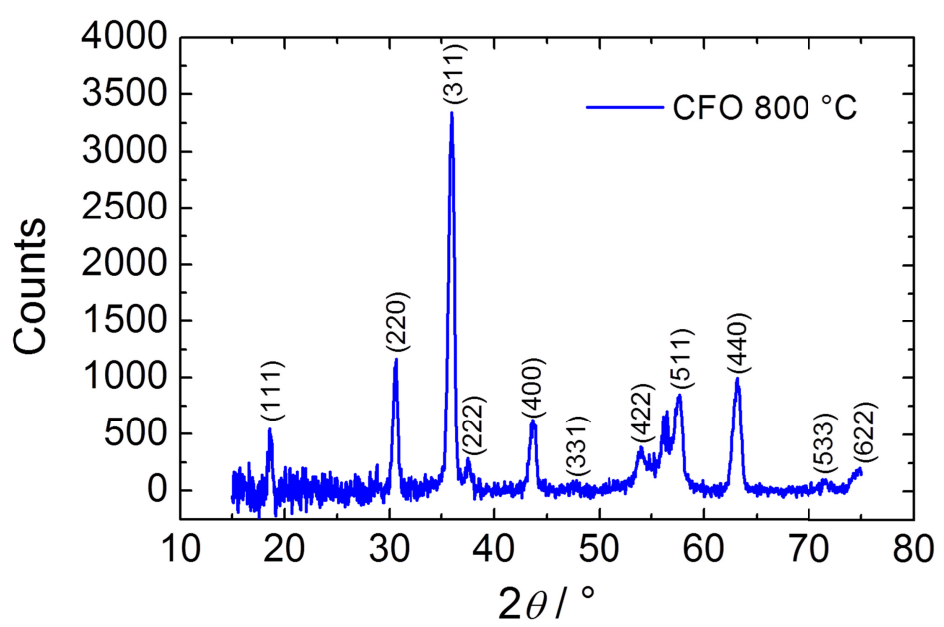

Figure S1. Grazing incidence XRD $2 \theta$ scan for the $\mathrm{CFO} 800{ }^{\circ} \mathrm{C}$ film.

SQUID magnetometry verifies the large remanence and coercivity values found for the $800{ }^{\circ} \mathrm{C}$ annealed CFO sample by MOKE. From the similar out-of-plane and in-plane SQUID hysteresis loops, it can be concluded that the nanocrystalline CFO film shows no pronounced magnetic anisotropy. The same behavior was also observed for other sol-gel prepared CFO nanocrystals. ${ }^{44,45}$ The magnetic moment versus temperature scan shows a ferrimagnetic line shape with an extrapolated compensation temperature point at around $730 \pm 20 \mathrm{~K}$.
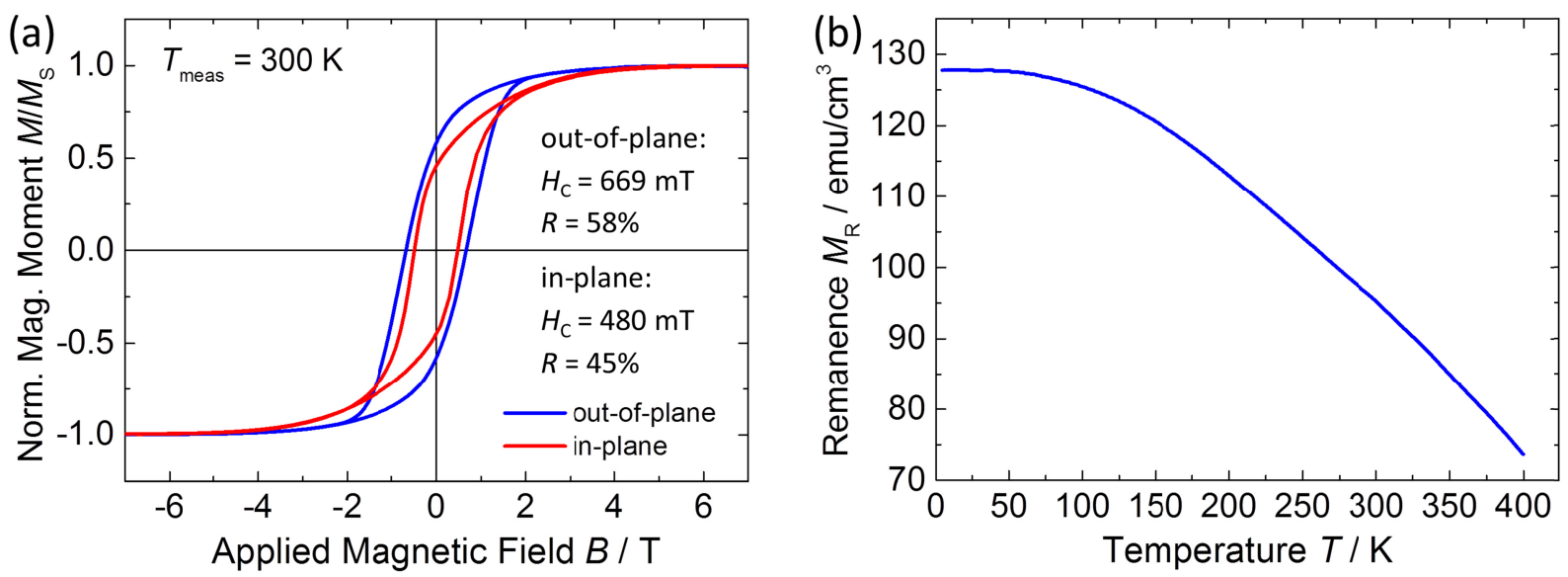

Figure S2. In-plane and out-of-plane SQUID hysteresis loop (a) and SQUID magnetic moment versus temperature scan (b) for $800{ }^{\circ} \mathrm{C}$ annealed CFO. 
The magnetic force microscopy image taken for a demagnetized $800{ }^{\circ} \mathrm{C}$ annealed $\mathrm{CFO}$ film shows a cluster-like structure similar to reference ${ }^{22}$ (where CFO films were prepared by PLD) with up and down out-of-plane magnetization components providing dark and bright contrast. Considering the average particle size of $33 \mathrm{~nm}$ as determined by AFM and TEM, it becomes clear that there are no magnetically independent single domain CFO nanoparticles, e.g. no Stoner-Wohlfarth behavior. Instead, the large observable magnetic clusters consist of several tens to hundreds of CFO nanoparticles with parallel magnetization.

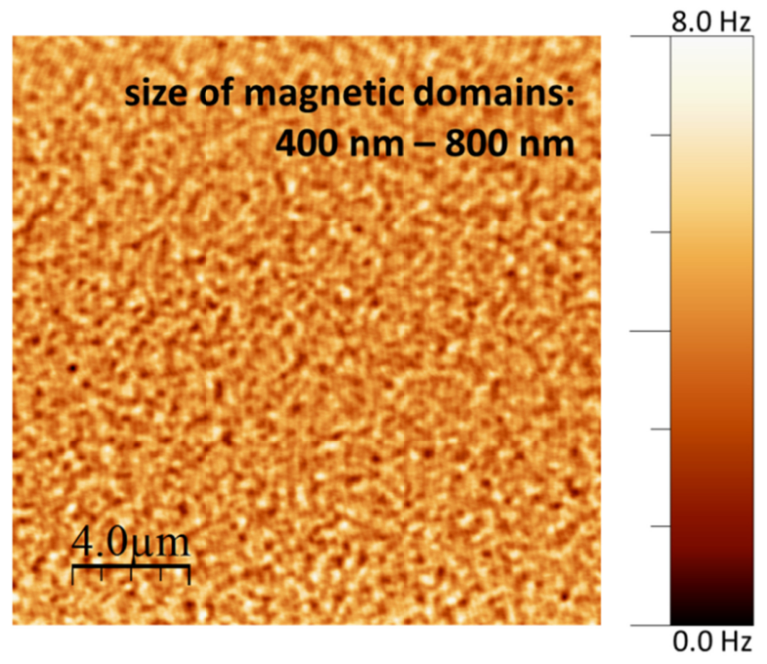

Figure $S_{3}$. MFM image for $800{ }^{\circ} \mathrm{C}$ annealed $\mathrm{CFO}$.

The MOKE hysteresis loops recorded for other ferrite materials illustrate the great versatility of the spin coating procedure for engineering magnetic oxides. When doping CFO with $5 \%$ of $\mathrm{Zn}^{2+}$ ions, the width of the magnetic hysteresis is decreased, but the typical trend for inverse spinels of improving magnetism with increasing annealing temperature is maintained. Pure $\mathrm{NiFe}_{2} \mathrm{O}_{4}$, also an inverse spinel, shows a narrow hysteresis loop that is also more pronounced for high annealing temperatures. In contrast, $\mathrm{ZnFe}_{2} \mathrm{O}_{4}$ behaves antiferromagnetically when annealing leads to good crystalline quality, while the low temperature annealed film shows defect-induced ferromagnetism. ${ }^{97}$
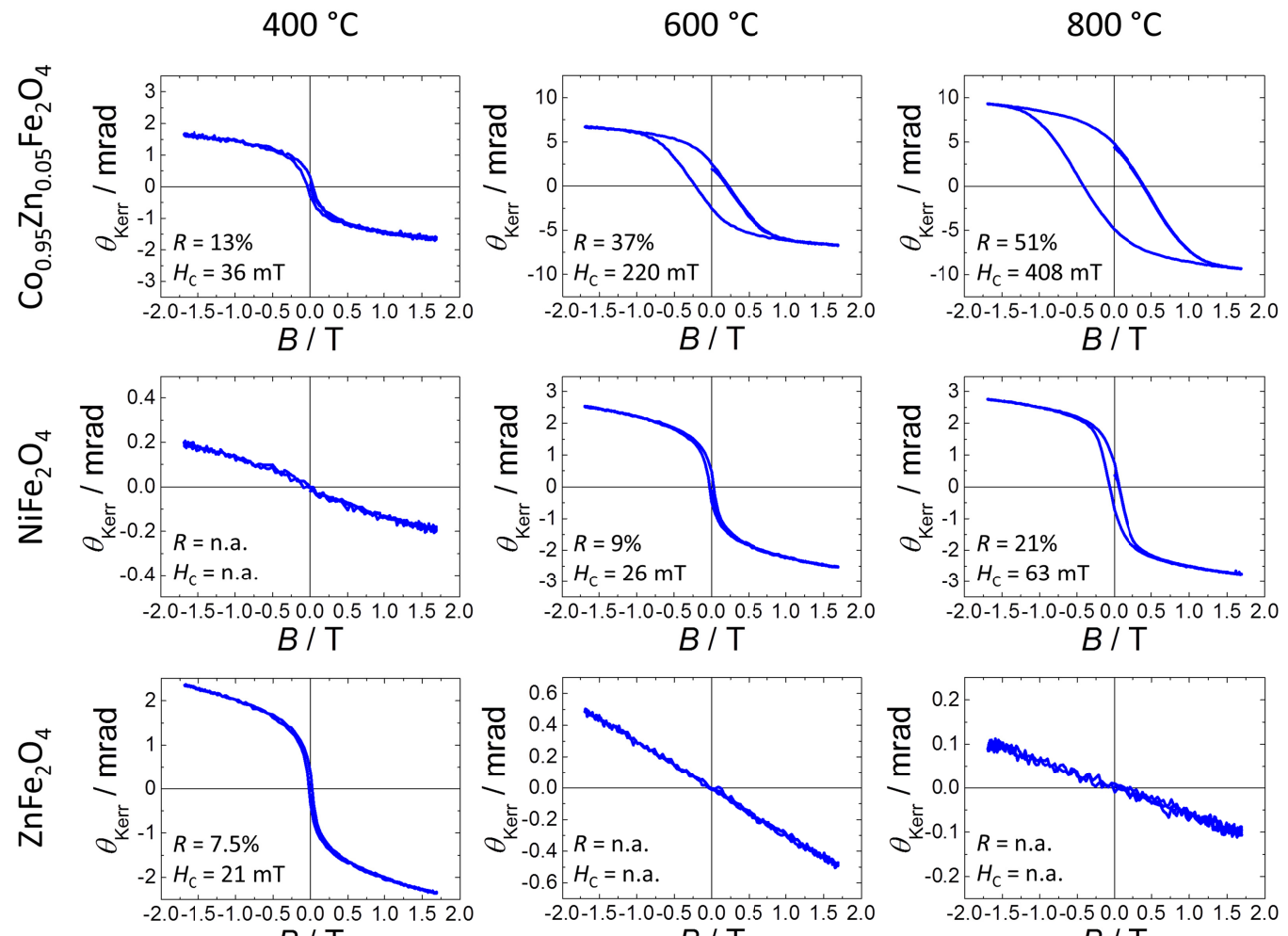

$B / \mathrm{T}$

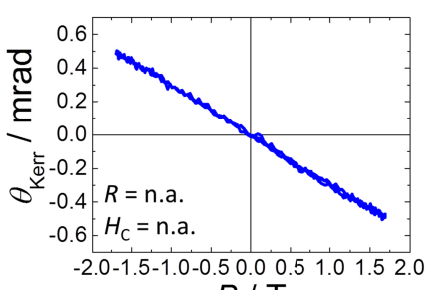

$$
B / \mathrm{T}
$$

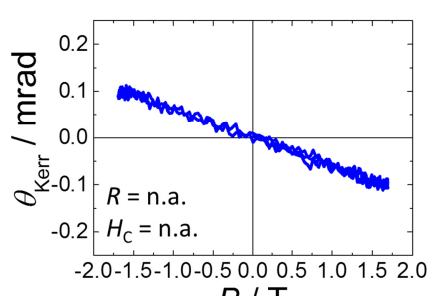

Figure S4. MOKE hysteresis curves for CZFO, NFO and ZFO. 